\title{
Reactive Pad-Batch Dyeing in CORONA Discharged Fabrics
}

Carneiro, N.; Souto, A.P.; Nogueira, C.

\begin{abstract}
The present work aims to the study of the increase of pick-up, together with uniformity degree, concerning the application of reactive dyeing baths by padding in a dry cotton fabric previously treated with CORONA discharge. Dyeing recipes, with and without wetting agent, are compared and advantages of CORONA discharge are analysed. Reactive dyes are used, expecting also to increase washing fastness of the difficult dark colours due to higher penetration of the dye into the core of the fibres when submitted to plasmatic treatment. The use of CORONA discharge intends to modify hydrophility of cotton fabrics even when they are presented to padding in hydrophobic state. A good alternative to wetting agent presence in recipes is found and more uniform and intense dyeings are performed in all cases.
\end{abstract}

